\title{
PRIVACY-PRESERVING IN MULTI-PARTY DATA RELEASE ON CLOUD FOR BIG DATA USING FUSION LEARNING
}

\author{
Divya Dangi ${ }^{1}$, Dr. G. Santhi ${ }^{2}$ \\ ${ }^{1}$ Ph.D. Research Scholar, Department of Computer Science and Application, Sarvepalli Radhakrishnan University, NH 12, RKDF \\ IST Campus, Hoshangabad Road, Misrod, Bhopal (M.P.) \\ ${ }^{2}$ Associate Professor, Department of Computer Science and Application, Sarvepalli Radhakrishnan University, NH 12, RKDF IST \\ Campus, Hoshangabad Road, Misrod, Bhopal (M.P.)
}

\begin{abstract}
The data volume increases every day and the next wave of apps cannot be envisaged without the created and executed data-driven algorithms. In this post, we undertook an extensive survey on privacy issues in the context of big data. At every point of the Big Data life cycle, we explored privacy challenges and discussed some of the advantages and disadvantages of the Big Data application of new privacy conservation schemes. Much progress has been made in protecting the protection of consumers from data production to data storage, but many transparent questions and hurdles exist.
\end{abstract}

\section{Keywords: Big Data, Multi-Party , Privacy-Preserving..}

\section{Introduction}

As we live in a time with massive data, we can easily generate and capture high-speed data with a vast spectrum of data that can differ in veracity (e.g. accurate data, imprecise and unclear data). Valuable knowledge and valuable information, where broad data science solutions can be identified, are contained in these big data. Daily model mining seeks to uncover implicit, secret and potentially valuable information and knowledge in a collection of frequently co-occurring items and/or events as a typical data science practise.

In order to find frequent models from accurate data, many existing pattern mining algorithms use a transaction-centric mining system. However, conditions are more suited for an item-centered mining approach, while cases are also more inaccurate and volatile. Therefore, in this post, we are implementing an object-centered algorithm for the mining of frequent patterns from large unknown info. In recent years, the scientific group has gained attention from significant numbers, based on similar technological advances (e.g. clouds) and modern paradigms (e.g., social networks). As large data is usually digitally available to assist with knowledge processing and performance processes, several owners of theoretically safe multi-part computing issues generally approach these huge data. These are the results.

The security and protection of large data has now become a core concern. This paper not only provides an item-centered algorithm for the mining of frequent patterns from largely unknown data. In other terms, in this post, we are implementing an item-centric algorithm to preserve our privacy in order to derive recurring patterns from a vast variety of unknown data. The results of our theoretical and empiric analysis suggest that our algorithm works in anonymity, preserving the usefulness of daily patterns from a broad variety of unknown details.

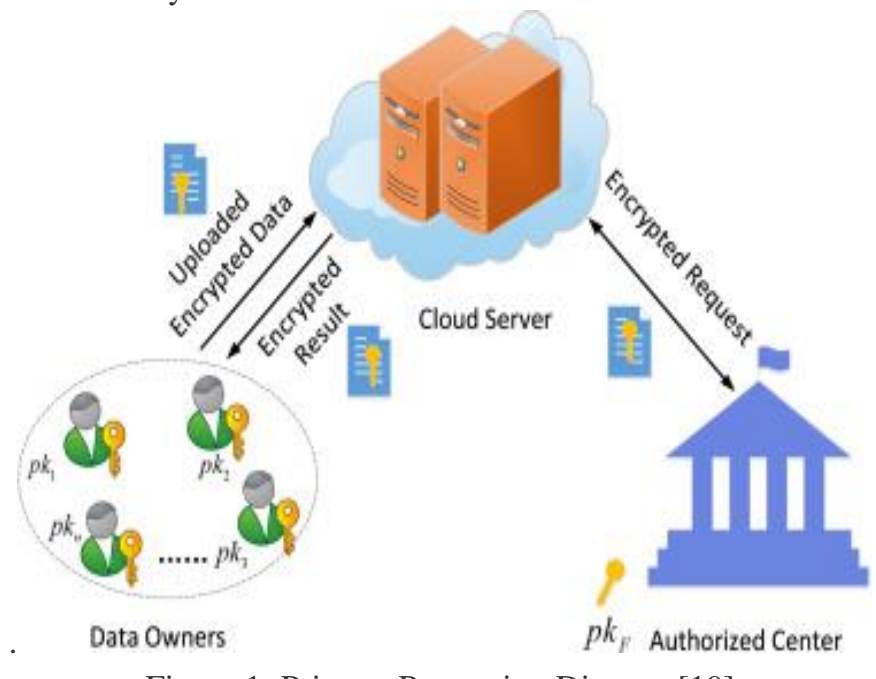

Figure 1: Privacy-Preserving Diagram[19]

It would not be appropriate to provide a dynamic distributed infrastructure. We build an ex post data processing method to calculate potential privacy threats. We use KL divergence calculations and Chebyshev inequalities to identify statistical flaws in the predicted privacy losses for a provided dataset. In this post, we shall make the following contributions:

- We suggest a modern yet easy approach to private data exchange, and this is the first realistic alternative to complicated real-world data to the best of our knowledge.

- We present a modern mathematical method for calculating future data breach privacy losses.

- Our methodology shows that model release approaches attain learning performance and are compatible with the reverse attack model. 


\section{Related Work}

The naive solution is for the data owner to disguise the meanings of the item in his transaction database by replacing objects with integrals in order to protect the privacy of the company and allow the server to comply with the association rules on data mining in the cloud (where the same item is replaced with the same integer while different items are replaced with different integers). This one-to-one replacement technique does not cover the frequency of the object. If the server has correct information on the occurrence of certain artefacts, it may re-identify certain things, especially the more common ones. Suppose, for example, that bread is the most prominent thing in retail transaction databases and that the integer is most commonly contained in the transformed database, the server may assume that the integer is bread.

(C. K. Leung et al, 2018) Paper-a privacy protection-centered element-centered algorithm that produces frequent patterns from largely unknown data.

(Y. Canbay et al.,2018) This research explores the data protection problem of big data, examines the big data aspects in the publication of vast data from a safety point of view, the challenges to privacy and data security. Finally, the results were analysed and explored and new proposals were raised.

(A. Cuzzocrea et al., 2018) provide applicable application of the above system, the data-dRIven aggregate-DRIFPROvenance Major Multidimensional Knowledge (DRIPROM), which takes multidimensional data directly into account as an interest.

(N. Gruschka, et al,2018) What types of knowledge may become a threat to privacy, the techniques used to defend privacy and their impact on the collection of data and the results of the analysis in accordance with the legal requirements.

(A. Cuzzocrea et al, 2020) To have an in-depth experimental and evaluational contribution to a state-of-the-art, distributed OLAP data security system, called SPPOLAP, whose principal advantage is the implementation of a whole new OLAP data cube data privacy concept.

(A. Heifetz et al., 2017) In particular, when running on data sets that are tiny enough to fit into memory, the production of Shade is dramatically higher than that of older differential privacy systems, and SparkSAM which often exceed the efficiency of the analogous non-private Spark query.

(S. Wang, et al.,2017) Show the feasibility of our suggested approach for large-scale social network placement datasets. Experimental results suggest that the POA mining approach can successfully affect Big Data scenarios while preserving the privacy of individual users.

P. Sreekumari (2018) This paper analyses existing current PPKS techniques on verifiability, consistency and data protection in a detailed manner. This research gives some valuable advice for future jobs.
I. V. Anikin(2017) The DBSCAN clustering algorithm implies data secrecy at all DBSCAN algorithm measures.

\section{Cloud Computing And Big Data}

Big data requires a massive amount of computation and storage, which means cloud infrastructure is required. Cloud computing allows businesses and corporations to adopt the cloud because of multiple advantages, such as cost savings and scalability. It also offers immense capacity for output and storage. Technologies such as virtualization, distributed storage and computing in cloud computing have made it easier to carry out practises that have been considered as daunting in conventional systems. However, computation may also lead to major concerns relating to privacy in the cloud. People are unwilling to transfer their personal or sensitive information to the cloud, except that the data is secured on the server. There are several hurdles to the creation of a secure and stable cloudbased huge data storage and processing infrastructure[7].

- Outsourcing: Today, companies prefer cloud outsourcing for their data to reduce their capital and operational costs. Outsourcing data to the cloud also means, though, that customers lose the control over their information. Lack of influence over performance is one of the main triggers of cloud instability. Insecurity would seriously breach the safety of cloud storage users. These concerns would be resolved by the secure operating device and data security. In addition, outsourced data in terms of security and completeness should also be verifiable to customers.

- Multi-tenancy: Virtualization has enabled many customers to share the same cloud service. Some space allocation policies can allow data belonging to separate cloud users to be stored in the same physical storage. Under such a case, access to details that do not belong to a malicious party is fairly clear. A number of issues, including data infringement and calculation infringement, can arise in such an environment. That is why it is absolutely important to establish mechanisms to cope with potential security and privacy risks.

- Massive computation: Because of the capacity of cloud networks for storing massive amounts of data collection and computing, traditional privacy mechanisms are inadequate.

\section{Approaches To Privacy Preservation Storage On Cloud}

Data security is largely three-dimensional, anonymous, integrity and availability as it is stored in the cloud[7]. The first two directly refer to data security, i.e. when data integrity or integrity has been infringed, the safety of the person has a significant effect. In this section, therefore, we would also discuss concerns of data integrity and fairness safety. The protection of a person's privacy is an essential requirement for the system for big data storage. Any mechanism to comply with this clause is in place. For eg, the sender may encrypt the data with a PKE such that the recipient can decrypt the data. 
As data is stored in the cloud, the following steps are taken to preserve the privacy of the user.

1) ATTRIBUTE BASED ENCRYPTION ABE [14],

[15] is An encryption programme that ensures an end to the anonymity of vast data in cloud computing. Data owner specify access policies in $\mathrm{ABE}$, and data is encrypted according to these policies. Only users whose characteristics comply with access policy established by data owner are able to decrypt the data. Data sharing protocols also have to be modified when coping with big data, as the data owner will be required to exchange them with various organisations. Access management systems focused on existing attributes [16], [17] do not take changes to regulation. The change of the strategy is a fantastic one.

2) IDENTITY BASED ENCRYPTION IBE is An option to PKE proposed to ease the use of human identity, using an e-mail address or an IP address as a public key in a certificate-based public key infrastructure (PKI). The IBE[19] scheme has been proposed to maintain anonymity between senders and receivers. The origins and purpose of the data can be protected secretly through the usage of these primitives. The IBE and ABE encryption scheme do not help updates to the ciphertext receiver. There are several ways for upgrading the ciphertext recipient. For eg, the data consumer will use the decryption and re-encoding mode. If the data is big, however, as with massive quantities, decryption and reencryption due to overhead computing can be time-consuming and costly. In addition, the data owner must remain online in this mode on an ongoing basis. Delegation to a reliable third party with a knowledge of the data owner's decryption key is another way to change the ciphertext recipients. This technique has some drawbacks, in as the system relies on the full confidence of a third party and the secrecy of the ciphertex recipient cannot be accomplished as the third party must accept the receiving of knowledge in order to begin the encryption method.

3) HOMOMORPHIC ENCRYPTION Multi-tenancy and virtualization make public cloud more susceptible to data security. In this case the probability of data loss will be very large and cloud participants occupy the same physical room. One approach to secure cloud data is to crypt and preserve data on the cloud, enabling computations of protected data to be carried out by the cloud. The form of encryption that enables functions to be measured on encrypted data is completely homomorphic[25]. Provided the encryption of a message, the encryption of a message function can only be achieved from the device on the encryption. Homorphic encryption offers complete secrecy but costs computing sophistication and is often quite challenging for current technology to enforce.

The release of confidential data to preserve the privacy of consumers is essential for a number of critical reasons, from health care to social sciences. Over the years, this issue has been addressed by proposing methods for the development of anonymisation and synthetic evidence. Unfortunately, strategies for anonymizing data do not guarantee strict confidentiality. While there are now synthetic data generation technologies that impose rigid definitions of differential privacy, these approaches are not, to our knowledge, extensively compared to a range of usability metrics. We're adding two new changes to this job. Next, various performance tests are used to evaluate existing approaches in several datasets. The second approach is a new one, combined with an efficient cost analysis of privacy, leveraging deep learning techniques to generate a range of synthetic data sets of greater data utility. We show that we can train deep learning models to catch correlations between different roles, and then use them to create distinctly private synthetic datasets. Our thorough experimental analysis of several data sets demonstrates that our system is superior (i.e. one of the highefficiency approaches to nearly all types of data that we have interacted with) than the state-of-the-art data utility approach.

Deep Generative Models A deep generative model represents the variables' distribution and is well suited to producing outputs of interest. Examples of the early versions of these models are Bayesian models and Helmholtz machines that mainly focus on unsupervised learning and latent space modeling. The generative model tries to understand the data distribution in an unsupervised fashion, i.e., a self-taught model. Well-trained generative models can synthesize new data similar to their training data. Since learning the exact data distribution is usually not practical, the alternative approach is to mimic the real data distribution pattern to represent the data. This approximation has been achieved utilizing deep neural networks, with impressive success. Recently, many researchers have paid attention to deep generative models, especially Generative Adversarial Networks (GANs) and Variational Autoencoders (VAEs). These have demonstrated remarkable improvements in a variety of domains, such as Computer Vision and NLP semi-supervised learning, and disentangled representation learning . Next, we describe one of the most commonly used generative models: Generative Adversarial Networks (GANs).

The generator learns the distribution pg from the real data distribution $\mathrm{x}$. We have $\mathrm{pz}(\mathrm{z})$ as the input noise prior, and the generator and discriminator functions are denoted as $\mathrm{G}(\mathrm{z} ; \theta \mathrm{g})$ and $\mathrm{D}(\mathrm{x} ; \theta \mathrm{d})$, respectively. $\mathrm{D}$ and $\mathrm{G}$ play the min-max game as follows:

$$
\begin{array}{r}
\underset{G}{\operatorname{Min}} \underset{D}{\operatorname{ax}} \mathcal{L}=E_{x \sim p_{d}(x)}[\log D(x)]+ \\
E_{z \sim p_{z}(z)}[1-\log D(G(z))]
\end{array}
$$

GANs are hard to train as many issues arise as the training proceeds, such as non-convergence and mode collapse.

Assume having an unconditional sample generation setting, with a dataset that is composed of samples $x$ (i). In 
unconditional sample generation, the labels y (i) do not play a role in image generation. However, we need the ground-truth labels to train our Siamese architecture. But, how and why? As explained earlier, a Siamese architecture utilizes two identical networks (networks with the same architectures and parameters $\theta$ ) to create a nonlinear mapping from its input domain to a shared Euclidean output feature space:

Proposed Algorithm

\section{Server executes:}

2 Initialize $\theta_{0}$

3 for $t=1$ to $T$ do

5

5

for each client $k \mathrm{~d} 0$

${ }_{6} \quad \theta_{t} \leftarrow \theta_{t-1}-\eta \sum_{k} g_{t}^{k} \quad \triangleright$ synchronized gradient updates

7

${ }_{8}$ ClientUpdate $(\theta)$ :

$9 \quad$ Select a batch $B$ from client's data

10 return local gradients $\nabla \mathcal{L}(B ; \theta)$

Due to the weight sharing and contrastive cost, such a scheme guarantees that:

- $\quad$ similar samples will stay close in the output feature

space.

- the model is robust against intra-class samples variations, as the model minimizes intra-class differences.

- $\quad$ dissimilar samples will simply be placed in distant places in the output space; and

- the model is robust against inter-class samples similarities as the model maximizes inter-class differences. Hence, we ideally will have separate clusters, in each of which there are samples from just one particular category. For example, all images belonging to dogs will be placed in one cluster, while pictures of cats will reside in another cluster in the output Euclidean space. Each cluster is a data manifold that belongs to that class. Such a learning paradigm will create a system that is able to:

- $\quad$ 1. Recognize the category of a fake sample, i.e., which cluster of data the fake sample belongs to (determining the closest cluster to classify the fake sample);

- 2. Determine how close the fake sample is to the cluster or any real data within (determining the quality of a fake sample given the real samples); and

- $\quad 3$. Clarify how diverse the generated fake samples are (to penalize the model for mode collapse).
To have an idea about novel tricks to train a GAN, please refer to .

We evaluate our method in two major ways.

We compare our results with various baseline methods:

- Deep Boltzmann Machines (DBMs): After training a stacked Deep Boltzmann Machine (DBM), we applied Gibbs sampling to create artificial data. Greedy contrastive divergence is used to build the model.

Variational Autoencoder (VAE): We employed VAEs : as one of the most used methods for generative purposes. 1D convolutional neural networks are utilized for both encoder and decoder.

First, we show that not only can deep education models be trained in the processing of data, but high learning performance can also be achieved. Second, we calculate analytical limitations on expected harm to privacy and assess the effectiveness of artificial data against reverse model attacks. For learning performance experiments, the following has been implemented:

1. Train a generative model (teacher) utilising just the testing break on the initial data collection.

2. Craete an artificial model dataset and use it to train ML models (students).

3. Assess students in the examination range. Notice that teacher-student models do not depend on each other. In addition, student modelling is not restricted to neural networks and can be used as a deep learning algorithm of any kind. We picked three standard image datasets for our experiments: MNIST, SVHN and CelebA. MNIST is a handwritten digit recognition dataset with 60000 training and 10,000 test cases; each example is in a grey image size of $28 \times 28$. SVHN is also a visual perception mission containing 73257 teaching images and 26032 studies. Samples of the house numbers in Google Street View are $32 \times 32$ pixel files. CelebA is a dataset of facial attributes containing 202599 pictures, 128x128 each, and $48 \times 48$ downscales.

\section{Conclusion}

The data fusion method of data mining, which includes security models, is a privacy-preserving technique. In this paper, we suggest a framework for the use of multi-party data fusion, where multiple parties store redundant attributes of a common group of individuals. In reality, the merged data is not vulnerable to contextual attacks or other conceptual attacks and may not have the characteristics of a human leaked. To do this, we present three anonymous and differential privacy algorithms. Experimental data sets demonstrate that the proposed algorithm can hold expertise of data mining operations effectively. 


\section{References}

[1] C. K. Leung, C. S. H. Hoi, A. G. M. Pazdor, B. H. Wodi and A. Cuzzocrea, "Privacy-Preserving Frequent Pattern Mining from Big Uncertain Data," 2018 IEEE International Conference on Big Data (Big Data), Seattle, WA, USA, 2018, pp. 51015110, doi: 10.1109/BigData.2018.8622260.

[2] Y. Canbay, Y. Vural and S. Sagiroglu, "Privacy Preserving Big Data Publishing," 2018 International Congress on Big Data, Deep Learning and Fighting Cyber Terrorism (IBIGDELFT), ANKARA, Turkey, 2018, pp. 24-29, doi: 10.1109/IBIGDELFT.2018.8625358.

[3] A. Cuzzocrea and E. Damiani, "Pedigree-ing Your Big Data: Data-Driven Big Data Privacy in Distributed Environments," 2018 18th IEEE/ACM International Symposium on Cluster, Cloud and Grid Computing (CCGRID), Washington, DC, 2018, pp. 675-681, doi: 10.1109/CCGRID.2018.00100.

[4] N. Gruschka, V. Mavroeidis, K. Vishi and M. Jensen, "Privacy Issues and Data Protection in Big Data: A Case Study Analysis under GDPR," 2018 IEEE International Conference on Big Data (Big Data), Seattle, WA, USA, 2018, pp. 5027-5033, doi: 10.1109/BigData.2018.8622621.

[5] A. Cuzzocrea, V. De Maio and E. Fadda, "Experimenting and Assessing a Distributed Privacy-Preserving OLAP over Big Data Framework: Principles, Practice, and Experiences," 2020 IEEE 44th Annual Computers, Software, and Applications Conference (COMPSAC), Madrid, Spain, 2020, pp. 1344-1350, doi: 10.1109/COMPSAC48688.2020.00-69.

[6] A. Heifetz, V. Mugunthan and L. Kagal, "Shade: A differentially-private wrapper for enterprise big data," 2017 IEEE International Conference on Big Data (Big Data), Boston, MA, 2017, pp. 1033-1042, doi: 10.1109/BigData.2017.8258027.

[7] S. Wang, R. Sinnott and S. Nepal, "Privacyprotected place of activity mining on big location data," 2017 IEEE International Conference on Big Data (Big Data), Boston, MA, 2017, pp. 1101-1108, doi: 10.1109/BigData.2017.8258035.

[8] P. Sreekumari, "Privacy-Preserving Keyword Search Schemes over Encrypted Cloud Data: An Extensive Analysis," 2018 IEEE 4th International Conference on Big Data Security on Cloud (BigDataSecurity), IEEE International Conference on High Performance and Smart Computing, (HPSC) and IEEE International Conference on Intelligent Data and Security (IDS), Omaha, NE,
2018, $\quad$ pp. 114-120, doi: 10.1109/BDS/HPSC/IDS18.2018.00035.

[9] I. V. Anikin and R. M. Gazimov, "Privacy preserving DBSCAN clustering algorithm for vertically partitioned data in distributed systems," 2017 International Siberian Conference on Control and Communications (SIBCON), Astana, 2017, pp. 1-4, doi: 10.1109/SIBCON.2017.7998473.

[10] A. Singh Rajawat and S. Jain, "Fusion Deep Learning Based on Back Propagation Neural Network for Personalization," 2nd International Conference on Data, Engineering and Applications (IDEA), Bhopal, India, 2020, pp. 1-7, doi: 10.1109/IDEA49133.2020.9170693.

[11] A. Mehmood, I. Natgunanathan, Y. Xiang, G. Hua and S. Guo, "Protection of Big Data Privacy," in IEEE Access, vol. 4, pp. 1821-1834, 2016, doi: 10.1109/ACCESS.2016.2558446.

[12] Z. A. Al-Odat and S. U. Khan, "Anonymous Privacy-Preserving Scheme for Big Data Over the Cloud," 2019 IEEE International Conference on Big Data (Big Data), Los Angeles, CA, USA, 2019, pp. 5711-5717, doi: 10.1109/BigData47090.2019.9006167.

[13] S. Sharma and A. S. Rajawat, "A secure privacy preservation model for vertically partitioned distributed data," 2016 International Conference on ICT in Business Industry \& Government (ICTBIG), Indore, 2016, pp. 1-6, doi: 10.1109/ICTBIG.2016.7892653.

[14] J. Jeon, J. Kim, J. Kim, K. Kim, A. Mohaisen and J. Kim, "Privacy-Preserving Deep Learning Computation for Geo-Distributed Medical Big-Data Platforms," 2019 49th Annual IEEE/IFIP International Conference on Dependable Systems and Networks - Supplemental Volume (DSN-S), Portland, OR, USA, 2019, pp. 3-4, doi: 10.1109/DSN-S.2019.00007.

[15] Nazmiye Ceren Abay, Yan Zhou, Murat Kantarcioglu, Bhavani Thuraisingham, and Latanya Sweeney. "Privacy Preserving Synthetic Data Release Using Deep Learning." Machine Learning and Knowledge Discovery in Databases (2018): 510-526.

[16] Martín Abadi, Andy Chu, Ian Goodfellow, H. Brendan McMahan, Ilya Mironov, Kunal Talwar, and Li Zhang. Deep learning with differential privacy. 2016. arXiv:1607.00133 [stat.ML].

[17] Nazmiye Ceren Abay, Yan Zhou, Murat Kantarcioglu, Bhavani Thuraisingham, and Latanya Sweeney. Privacy preserving synthetic data release using deep learning. In Joint European Conference 
on Machine Learning and Knowledge Discovery in Databases, pages 510-526. Springer, 2018.

[18] Gergely Acs, Luca Melis, Claude Castelluccia, and Emiliano De Cristofaro. Differentially private mixture of generative neural networks. IEEE Transactions on Knowledge and Data Engineering, 2018.

[19] S. Sharma and A. S. Rajawat, "A review of privacy preserving models for multiparty data release framework", Proc. ACM Symp. Women Res., pp. 165-168, 2016.

[20] A. S. Rajawat and A. R. Upadhyay, "Web Personalization Model Using Modified S3VM Algorithm For developing Recommendation Process," 2nd International Conference on Data, Engineering and Applications (IDEA), Bhopal, India, 2020, pp. 1-6, doi: 10.1109/IDEA49133.2020.9170701.

[21] PingLiaJinLiaZhenganHuangaTongLibChong-

ZhiGaoaSiu-MingYiuc, Multi-key privacypreserving deep learning in cloud computing, https://doi.org/10.1016/j.future.2017.02.006 .

[22] S. Sharma and A. S. Rajawat, "A secure privacy preservation model for vertically partitioned distributed data," 2016 International Conference on ICT in Business Industry \& Government (ICTBIG), Indore, 2016, pp. 1-6, doi: 10.1109/ICTBIG.2016.7892653.

[23] A. Singh Rajawat and S. Jain, "Fusion Deep Learning Based on Back Propagation Neural Network for Personalization," 2nd International Conference on Data, Engineering and Applications (IDEA), Bhopal, India, 2020, pp. 1-7, doi: 10.1109/IDEA49133.2020.9170693. 